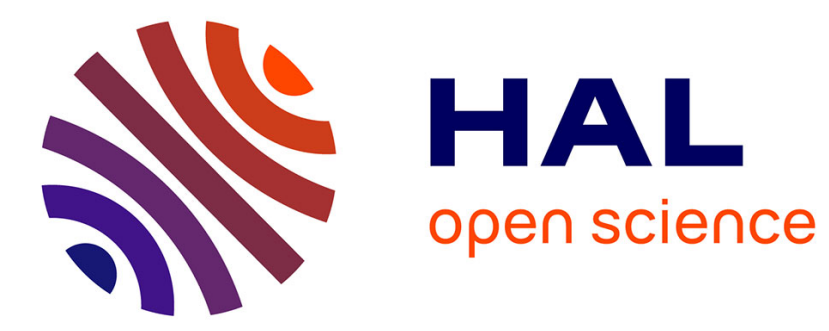

\title{
Minimum in the high-order harmonic generation spectrum from molecules: role of excited states Yong-Chang Han, Lars Bojer Madsen
}

\section{To cite this version:}

Yong-Chang Han, Lars Bojer Madsen. Minimum in the high-order harmonic generation spectrum from molecules: role of excited states. Journal of Physics B: Atomic, Molecular and Optical Physics, 2010, 43 (22), pp.225601. 10.1088/0953-4075/43/22/225601 . hal-00570211

\section{HAL Id: hal-00570211 \\ https://hal.science/hal-00570211}

Submitted on 28 Feb 2011

HAL is a multi-disciplinary open access archive for the deposit and dissemination of scientific research documents, whether they are published or not. The documents may come from teaching and research institutions in France or abroad, or from public or private research centers.
L'archive ouverte pluridisciplinaire HAL, est destinée au dépôt et à la diffusion de documents scientifiques de niveau recherche, publiés ou non, émanant des établissements d'enseignement et de recherche français ou étrangers, des laboratoires publics ou privés. 


\title{
Minimum in the high-order harmonic generation spectrum from molecules: Role of excited states
}

\author{
Yong-Chang Han and Lars Bojer Madsen
}

Lundbeck Foundation Theoretical Center for Quantum System Research, Department of Physics and Astronomy, Aarhus University, DK-8000 Aarhus C, Denmark

\begin{abstract}
We model the process of high-order harmonic generation by solving the time-dependent Schrödinger equation for $\mathrm{H}_{2}^{+}$in the fixed nuclei approximation including full 3D electron motion for nonvanishing angles between the nuclear axis and the linear polarization of the driving pulse. We show that the coherent laser coupling induced between the ${ }^{2} \Sigma_{g}^{+}\left(1 s \sigma_{g}\right)$ ground state and the first excited ${ }^{2} \Sigma_{u}^{+}\left(2 p \sigma_{u}\right)$ state leads to two dominating amplitudes for the high-order harmonic generation that may interfere: amplitudes describing recombination back into the $\sigma_{g}$ and $\sigma_{u}$ states, respectively. These two amplitudes may interference destructively or constructively. The effect of a destructive interference is very clear through the occurrence of a minimum in the high-order harmonic spectrum. We show cases where such a minimum in the spectrum is approximately at the position predicted by the simple two-center interference formula.
\end{abstract}

PACS numbers: 42.65.Ky, 32.80.Wr, 42.50.Hz

Submitted to: J. Phys. B: At. Mol. Phys. 


\section{Introduction}

Signatures of interference in terms of minima in high-order harmonic generation (HHG) spectra from small linear molecules has raised a lot of interest both theoretically and experimentally since structural and dynamical information may be retrived from the position of these minima (see, e.g., references [1-25]). In all these works, the interpretation of the origin of the minima is based on the three-step model $[26,27]$ or extensions therefore. The model describes how electrons that are released from the highest occupied molecular orbital, or from a few of the least bound valence shells $[11,15,20-25]$ in a tunnelling-like process, propagate freely in the external field, without influence from the molecular potential, and finally some $2 / 3$ of an optical period later, revisit the core and recombine into the original orbitals under the emission of radiation. Hence, the essential quantum mechanical ingredients in the model are the coherent addition of amplitudes describing the transition from initial orbitals into the Volkov continuum and back via the ionization and recombination dipole matrix elements. As we show in the present work, a minimum in the HHG spectrum may, however, also occur due to excited states, i.e., a property of the molecular electronic structure. The pump pulse used to produce HHG may transfer population from the ground to an excited state and the HHG process may now take place from the ground or the excited state, or more precisely will involve amplitudes describing recombination into the ground or the excited state. Since the harmonic signal is proportional to the norm square of the sum of amplitudes effects of constructive or destructive interference may influence the HHG spectrum and, for example, give rise to minima at particular internuclear distances and alignment angles. The influence of excited states has been investigated before for an initially prepared coherent superposition in the atomic case [28] and for $\mathrm{N}_{2}$ [29]. Here we address the problem fully dynamically, starting out in the ground state and focus on a minimum in the spectrum originating from the destructive interference between the amplitudes.

Minima in $\mathrm{HHG}$ spectra were first reported for $\mathrm{H}_{2}^{+}$and $\mathrm{H}_{2}$ in numerical investigations of the dependence on the molecular alignment with respect to the external field polarization $[1,2]$. The position of the minimum is dependent on the internuclear distance and orientation angle of the molecule $[2,4]$. The origin of the minimum was analyzed in the spirit of the three-step model [26, 27]. Assuming that most of the harmonics are generated by the recombination of the continuum electron wave packet into the initial ground state [27], and approximating the ground state by a linear combination of atomic orbitals, and the continuum electron wave packet by a plane wave, the minimum in the spectrum was shown to result from a destructive interference between the radiation emitted from the two nuclear centers. In this twocenter interference model $[2,4]$, the interference structure is related to the internuclear distance $R$, the angle $\beta$ between the molecular axis and the laser polarization direction, and also the symmetry of the molecular orbital. For a symmetric orbital, the two-center 
interference is governed by the term [2]

$$
I(\boldsymbol{k})=e^{-i \boldsymbol{k} \cdot \boldsymbol{R} / 2}+e^{i \boldsymbol{k} \cdot \boldsymbol{R} / 2}=2 \cos \left(\frac{1}{2} \boldsymbol{k} \cdot \boldsymbol{R}\right),
$$

and the two-center interference minima are expected to occur when the argument of the cosine in equation ( 1 ) is an odd multiple of $\pi / 2$, i.e., following the formula (here and throughout atomic units are used)

$$
\begin{aligned}
& k R \cos \beta=(2 n+1) \pi, \quad n=0,1,2, \ldots, \\
& N_{\min }^{\mathrm{ti}} \omega_{0}=k^{2} / 2,
\end{aligned}
$$

where $k$ is the electron momentum, $N_{\min }^{\mathrm{ti}}$ is the harmonic order at the two-center interference minimum and $\omega_{0}$ the center frequency of the laser field. Because recombination occurs near the nuclei and the electron is approximated by a plane wave, the electron momentum $k$ in equation (2) accounts for the effect of the Coulomb potential $[2,4,7]$. The two-center interference model was, for example, used to explain the minima in the $\mathrm{HHG}$ spectrum of $\mathrm{H}_{2}^{+}$and $\mathrm{H}_{2}$ calculated by the time-dependent Schrödinger equation (TDSE) $[2,4]$ and the model was extended to the dynamic twocenter interference taking into consideration the attosecond nuclear motion [17]. The two-center interference minimum relates directly to the internuclear distance and the symmetry of the highest occupied molecular orbital (HOMO) of the molecule.

In addition to the effects relating to the HOMO, it was found for multi-electron molecules that states lying energetically below the HOMO can contribute to the HHG spectrum [11,15,20-25,30,31]. Hence, measurement [15] and theory [11] of the alignment dependence of the $\mathrm{HHG}$ spectrum of $\mathrm{N}_{2}$ have showed that the second least bound orbital, the HOMO-1, can also influence the spectrum. More recently, both the HOMO and the HOMO-1 of $\mathrm{N}_{2}$ have been reconstructed tomographically using HHG [23]. Moreover, studies on the HHG spectrum of $\mathrm{CO}_{2}$ have demonstrated that the HOMO-2 also influences the HHG spectrum [20-22, 24, 25]. Multiple orbitals provide different channels for the processes of ionization and recombination, whose relative phases and amplitudes lead to interference. A minimum in the HHG spectrum of $\mathrm{CO}_{2}$ can be associated with an interference between the $\mathrm{HOMO}$ and $\mathrm{HOMO}-2$, which suggests that multi-electron dynamics in molecules can be imaged from the HHG spectrum [21, 22]. Note that earlier work $[13,14,18]$ ascribed the minimum in the HHG spectrum of $\mathrm{CO}_{2}$ to the two-center interference effect discussed above, i.e., a single-electron mechanism. The observation that the minimum changes its position for varying wavelength and intensity $[24,25]$ clearly shows that it has a different origin than solely the two-center interference effect.

In this work, we demonstrate yet another mechanism that may lead to a minimum in the HHG spectrum. We show that the electronic states lying energetically above the HOMO can also influence the HHG spectrum and interference between the amplitudes describing recombination into excited states and into the $\mathrm{HOMO}$, respectively, may give rise to a minimum in the HHG spectrum. This is a surprising effect since HHG is normally understood to be completely dominated by recombination back into the initial 
state (HOMO). As an example illustrating the effect, we consider the $\mathrm{H}_{2}^{+}$molecule and perform 3D time-dependent Schrödinger equation (TDSE) calculations for fixed internuclear distance and an alignment angle $\beta \neq 0$. We analyze the total HHG spectrum $S_{\text {tot }}(\omega)$ in terms of the components $S_{\mathrm{g}}(\omega)$ araising from the recombination into the ${ }^{2} \Sigma_{g}^{+}\left(1 s \sigma_{g}\right)$ ground state (in short $\left.\sigma_{g}\right)$ and $S_{\mathrm{u}}(\omega)$ from the recombination into the ${ }^{2} \Sigma_{u}^{+}\left(2 p \sigma_{u}\right)$ excited state (in short $\sigma_{u}$ ) and also the interference term from the two amplitudes.

\section{Theory and discussion}

The total wave function $\psi_{\text {tot }}(\boldsymbol{r}, t)$ is calculated in the length gauge by solving the TDSE using a grid method for the radial coordinate and a representation in terms of spherical harmonics, $Y_{l m}(\theta, \phi)$, for the angular part $[32,33]$. The linearly polarized external pulse has a trapezoidal envelope with a total duration of 7 optical cycles and linear ramps of 1.5 optical cycles. The calculations were performed at $800 \mathrm{~nm}$ and peak intensities of $1.7 \times 10^{14} \mathrm{~W} / \mathrm{cm}^{2}$ and $4.0 \times 10^{14} \mathrm{~W} / \mathrm{cm}^{2}$. We use an equidistant grid with 2048 points that extends up to 90 Bohr. The angular basis set contains 41 spherical harmonics. The calculations were repeated in a larger box (125 Bohr with 3072 grid points) with a larger angular basis (46 spherical harmonics), and the results are converged. We note that for nonparallel geometries, $\beta \neq 0$, there is no azimuthal symmetry, and the problem is fully 3D, so, e.g., for each $l$ we have $2 l+1$ values of $m$. Our method, including a comparison of the computational efficiency in length and velocity gauges, is described in reference [34].

The HHG spectrum is calculated by the absolute square of the Fourier transform of the time-dependent dipole acceleration [35].

$$
S_{\text {tot }}^{\hat{e}}(\omega)=\left|\int \hat{\boldsymbol{e}} \cdot\left\{\left\langle\psi_{\text {tot }}(\boldsymbol{r}, t)|\nabla V(\boldsymbol{r})| \psi_{\text {tot }}(\boldsymbol{r}, t)\right\rangle+\boldsymbol{E}(t)\right\} e^{i \omega t} d t\right|^{2} .
$$

Here $V(\boldsymbol{r})$ and $\boldsymbol{E}(t)$ are the molecular potential and the electric field, respectively. We consider the spectrum emitted along the laser polarization direction, i.e., the direction of the unit vector $\hat{\boldsymbol{e}}$ in equation (3) is parallel to the laser polarization direction (see reference [36] and references therein for a discussion of the perpendicular component). The total wave function describes fully coherently the electron wavepacket generated by the external field. For the following discussion is it advantageous to represent this wavepacket in an expansion accounting explicitly for the two lowest-lying field-free bound states $\psi_{\text {tot }}(\boldsymbol{r}, t)=\psi_{\mathrm{g}}(\boldsymbol{r}, t)+\psi_{\mathrm{u}}(\boldsymbol{r}, t)+\psi_{\text {res }}(\boldsymbol{r}, t)$. In this expression $\psi_{\mathrm{g}(\mathrm{u})}(\boldsymbol{r}, t)=$ $c_{\mathrm{g}(\mathrm{u})}(t) \psi_{\mathrm{g}(\mathrm{u})}(\boldsymbol{r})$ with $\psi_{\mathrm{g}(\mathrm{u})}(\boldsymbol{r})$ the $\sigma_{\mathrm{g}}$ ground $\left(\sigma_{\mathrm{u}}\right.$ first excited) state wavefunction and $c_{\mathrm{g}(\mathrm{u})}(t)$ are the time-dependent expansion coefficients obtained by projection on the total wavepacket. In the description of the total wavepacket, $\psi_{\text {tot }}(\boldsymbol{r}, t)$, the part $\psi_{\text {res }}(\boldsymbol{r}, t)$ denotes the rest of the wavepacket, i.e., the part that is not in $\sigma_{g}$ or $\sigma_{u}$, and which in an expansion on field-free eigenstates populates many excited bound states and the continuum up to a characteristic cut-off depending on the laser parameters. By inserting 
the expression for the total wave packet into equation (3), we obtain

$$
\begin{aligned}
S_{\mathrm{tot}}(\omega) & \approx\left|A_{\mathrm{g}}(\omega)+A_{\mathrm{u}}(\omega)\right|^{2} \\
& =S_{\mathrm{g}}(\omega)+S_{\mathrm{u}}(\omega)+2 \operatorname{Re}\left[A_{\mathrm{g}}(\omega) A_{\mathrm{u}}^{*}(\omega)\right],
\end{aligned}
$$

with

$$
\begin{aligned}
& A_{\xi}(\omega)=\int 2 \operatorname{Re}\left[\left\langle\psi_{\xi}(\boldsymbol{r}, t)|\nabla V(\boldsymbol{r})| \psi_{\text {res }}(\boldsymbol{r}, t)\right\rangle\right] e^{i \omega t} d t \\
& S_{\xi}(\omega)=\left|A_{\xi}(\omega)\right|^{2}, \quad \xi=\mathrm{g}, \mathrm{u} .
\end{aligned}
$$

In equation (3), we neglect the Fourier transform of the external field $E(t)$, and the Fourier transform of the coupling between the two bound states, $2 \operatorname{Re}\left[\left\langle\psi_{\mathrm{g}}(\boldsymbol{r}, t)|\nabla V(\boldsymbol{r})| \psi_{\mathrm{u}}(\boldsymbol{r}, t)\right\rangle\right]$, because these two terms only contribute to the lower orders of the spectrum that are not of interest for the present study. In equation (4) we also neglect the Fourier transform of $\left\langle\psi_{\text {res }}(\boldsymbol{r}, t)|\nabla V(\boldsymbol{r})| \psi_{\text {res }}(\boldsymbol{r}, t)\right\rangle$, which, we have checked, has a very small contribution because the recombination into the higher excited states and the continuum is very weak. Thus, equation (4) represents what we have found from our TDSE calculations: the total HHG spectrum mainly consists of terms describing the recombination into the initial ground state $S_{\mathrm{g}}(\omega)$, the recombination into the first excited state $S_{\mathrm{u}}(\omega)$, and the interference term $2 \operatorname{Re}\left[A_{\mathrm{g}}(\omega) A_{\mathrm{u}}^{*}(\omega)\right]$. In the following, we will refer to the interference term as the orbital interference term, and we will demonstrate that both the terms describing recombination into the excited state and the orbital interference term between the $\sigma_{g}$ and $\sigma_{u}$ states play important roles in the formation of the total spectrum.

The different components of the HHG spectrum are shown in figure 1 for the internuclear distance fixed at the equilibrium $R=2.0$ Bohr. The laser peak intensity is $4 \times 10^{14} \mathrm{~W} / \mathrm{cm}^{2}$, and the angle $\beta$ between the molecular axis and the laser polarization direction is $\beta=20^{\circ}$. In figure 1 (a) the total HHG spectrum $S_{\text {tot }}(\omega)$, which contains the orbital interference term $2 \operatorname{Re}\left[A_{\mathrm{g}}(\omega) A_{\mathrm{u}}^{*}(\omega)\right]$ according to equation (4) is shown as the solid (black) curve, and the sum $S_{\mathrm{g}}(\omega)+S_{\mathrm{u}}(\omega)$ by the dashed (red) curve. The two spectra are on top of each other in most parts of the spectrum, showing that the interference term is typically negligible. At harmonic order 21 , however, we find a dip in the total spectrum $S_{\text {tot }}(\omega)$. Since this dip is not present in the $S_{\mathrm{g}}(\omega)+S_{\mathrm{u}}(\omega)$ result, it is caused by the orbital interference term included in $S_{\text {tot }}(\omega)$. In order to obtain a good estimate of the minimum position and to make a detailed analysis of the HHG spectrum, we calculated the smoothed spectra, $\int S(\widetilde{\omega}) \exp \left(-(\widetilde{\omega}-\omega)^{2} / \sigma^{2}\right) d \widetilde{\omega}$ with $\sigma=3 \omega_{0}$ [2]. In figure 1(b), the dotted (blue) curve and dash-dotted (green) curve are the spectra associated with recombination into the $\sigma_{\mathrm{g}}$ ground state $S_{\mathrm{g}}(\omega)$ and into the $\sigma_{\mathrm{u}}$ first excited state $S_{\mathrm{u}}(\omega)$, respectively. It can be seen that there is a minimum in $S_{\mathrm{g}}(\omega)$, as pointed to by the dotted (blue) arrow at harmonic order 25, which is in good agreement with the prediction of the two-center interference model equation (2). In figure 1(c), we present a zoom-in of the region of the interest.

To analyse this situation more closely, we first note that for the excited $\sigma_{\mathrm{u}}$ state, which is an antisymmetric orbital, the two-center interference condition of equation (1) 

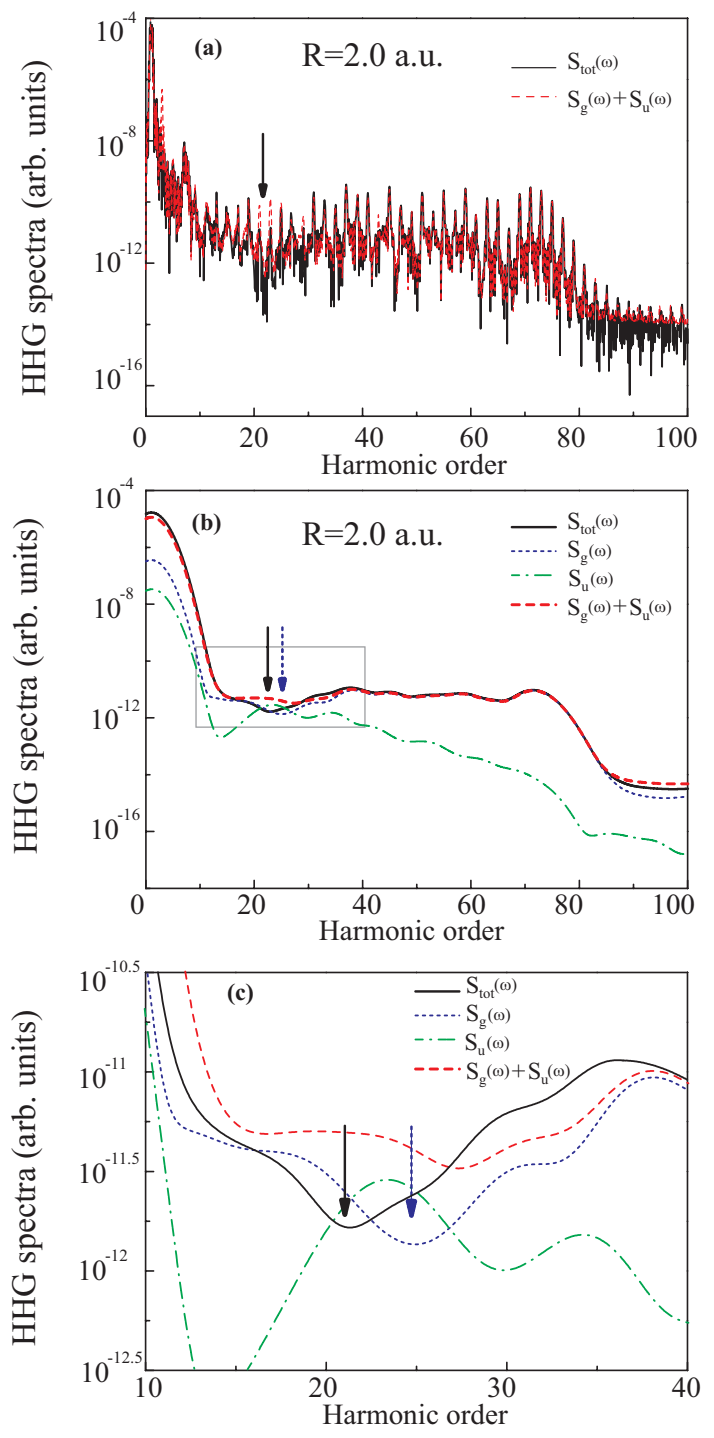

Figure 1. HHG spectrum of $\mathrm{H}_{2}^{+}$for internuclear distance $R=2.0 \mathrm{Bohr}$, alignment angle between the linear polarization and the internuclear axis $\beta=20^{\circ}, I=4 \times 10^{14}$ $\mathrm{W} / \mathrm{cm}^{2}, 800 \mathrm{~nm}$, and 7 optical cycles. (a) Total HHG spectrum $S_{\text {tot }}(\omega)$ [solid (black) curve] including the orbital interference term, and the sum $S_{\mathrm{g}}(\omega)+S_{\mathrm{u}}(\omega)$ [dashed (red) curve] without the orbital interference term. (b) Smoothed spectrum components. The total HHG spectrum $S_{\text {tot }}(\omega)$ [solid (black) curve], the component $S_{\mathrm{g}}(\omega)$ from $\sigma_{\mathrm{g}}$ [dotted (blue) curve], the component $S_{\mathrm{u}}(\omega)$ from $\sigma_{\mathrm{u}}$ [dash-dotted (green) curve], and the sum $S_{\mathrm{g}}(\omega)+S_{\mathrm{u}}(\omega)$ [dashed (red) curve]. The solid (black) arrow points to the orbital interference minimum in $S_{\text {tot }}(\omega)$ and the dashed (blue) arrow points to the two-center interference minimum associated with the recombination into the ground state, equation (2). (c) Zoom-in of (b). 
changes to $[4]$

$$
I(\boldsymbol{k})=e^{-i \boldsymbol{k} \cdot \boldsymbol{R} / 2}-e^{i \boldsymbol{k} \cdot \boldsymbol{R} / 2}=-2 i \sin \left(\frac{1}{2} \boldsymbol{k} \cdot \boldsymbol{R}\right) .
$$

Thus, the component $S_{\mathrm{u}}(\omega)$ will show maxima at the harmonic order $N_{\text {min }}^{\mathrm{ti}}$ obtained by equation (2), where the component $S_{\mathrm{g}}(\omega)$ shows minima. As the dash-dotted (green) curve shows in figures $1(\mathrm{~b})$-(c), the signal of $S_{\mathrm{u}}(\omega)$ in the vicinity of $N_{\text {min }}^{\mathrm{ti}}=25$ is indeed a little stronger than $S_{\mathrm{g}}(\omega)$. This difference occurs because the antisymmetric orbital structure of the $\sigma_{\mathrm{u}}$ state leads to constructive two-center interference at $N_{\text {min }}^{\mathrm{ti}}=25$ for $S_{\mathrm{u}}(\omega)$. As a result, the sum $S_{\mathrm{g}}(\omega)+S_{\mathrm{u}}(\omega)$ as shown by the dashed (red) curve does not represent the two-center interference minimum at $N_{\min }^{\mathrm{ti}}=25$ due to the effect of $S_{\mathrm{u}}(\omega)$. However, the total spectrum $S_{\text {tot }}(\omega)$ [solid (black) curve], which contains the interference term $2 \operatorname{Re}\left[A_{\mathrm{g}}(\omega) A_{\mathrm{u}}^{*}(\omega)\right]$, shows a minimum at harmonic order 21 as pointed to by the solid (black) arrow. Comparing $S_{\text {tot }}(\omega)$ with $S_{\mathrm{g}}(\omega)+S_{\mathrm{u}}(\omega)$, we see that the minimum in the total HHG spectrum is not caused by the two-center interference, but instead caused by the orbital interference between the initial $\sigma_{\mathrm{g}}$ ground and $\sigma_{\mathrm{u}}$ first excited states. At $R=2.0 \mathrm{Bohr}$, the coupling between these two bound states is weak, which leads to a relatively small magnitude of $S_{\mathrm{u}}(\omega)$. Because the destructive two-center interference associated with recombination into $\sigma_{\mathrm{g}}(\omega)$ and the constructive two-center interference associated with recombination into $\sigma_{\mathrm{u}}(\omega)$ occur around the same harmonic order $N_{\min }^{\mathrm{ti}}$ as predicted by equation (2), the contributions from the two components are comparable and hence the orbital interference term $2 \operatorname{Re}\left[A_{\mathrm{g}}(\omega) A_{\mathrm{u}}^{*}(\omega)\right]$ contributes considerably to the total spectrum. As a result, the orbital interference leads to the minimum in the total HHG spectrum. The orbital interference term may also give rise to an increase in the HHG signal due to constructive interference. This is observed in figure $1(\mathrm{c})$, where the total signal is higher than the sum of signals from recombination into the $\sigma_{g}$ and $\sigma_{u}$ states for harmonics between $\sim 27-39$.

For larger internuclear distances, the orbital interference minimum can take place at smaller laser intensity, since the transition dipole moment between the $\sigma_{\mathrm{g}}$ and $\sigma_{\mathrm{u}}$ states increases with $R$ [37]. In figure 2, the HHG spectrum is shown for $R=3.0$ Bohr, peak intensity $I=1.7 \times 10^{14} \mathrm{~W} / \mathrm{cm}^{2}$, and alignment angle $\beta=55^{\circ}$. In figure $2(\mathrm{a})$, no minimum structure can be found in the sum $S_{\mathrm{g}}(\omega)+S_{\mathrm{u}}(\omega)$ [the dashed (red) curve], which does not include the orbital interference term. When we take the orbital interference into account, i.e., consider the total HHG spectrum $S_{\text {tot }}(\omega)$ as shown by the solid (black) curve, a minimum is observed around harmonic order 33, pointed to by the solid (black) arrow. As shown in figure 3, although the laser intensity used in figure 2 is weaker than that used in figure 1, the temporary population of the $\sigma_{\mathrm{u}}$ state for $R=3.0$ Bohr is larger than that at the equilibrium, which means, due to the smaller binding energy of the $\sigma_{u}$ state, that more population can be transferred into the continuum and later recombine. As a result, at $R=3.0 \mathrm{Bohr}$, the magnitude of $S_{\mathrm{u}}(\omega)$ increases significantly. As shown by the smoothed spectrum components in figure $2(\mathrm{~b}), S_{\mathrm{u}}(\omega)$ is stronger than $S_{\mathrm{g}}(\omega)$ for the harmonic orders $30 \sim 40$. According to the two-center interference formula equation (2), for $R=3.0 \mathrm{Bohr}$ and $\beta=55^{\circ}$, the HHG spectrum 

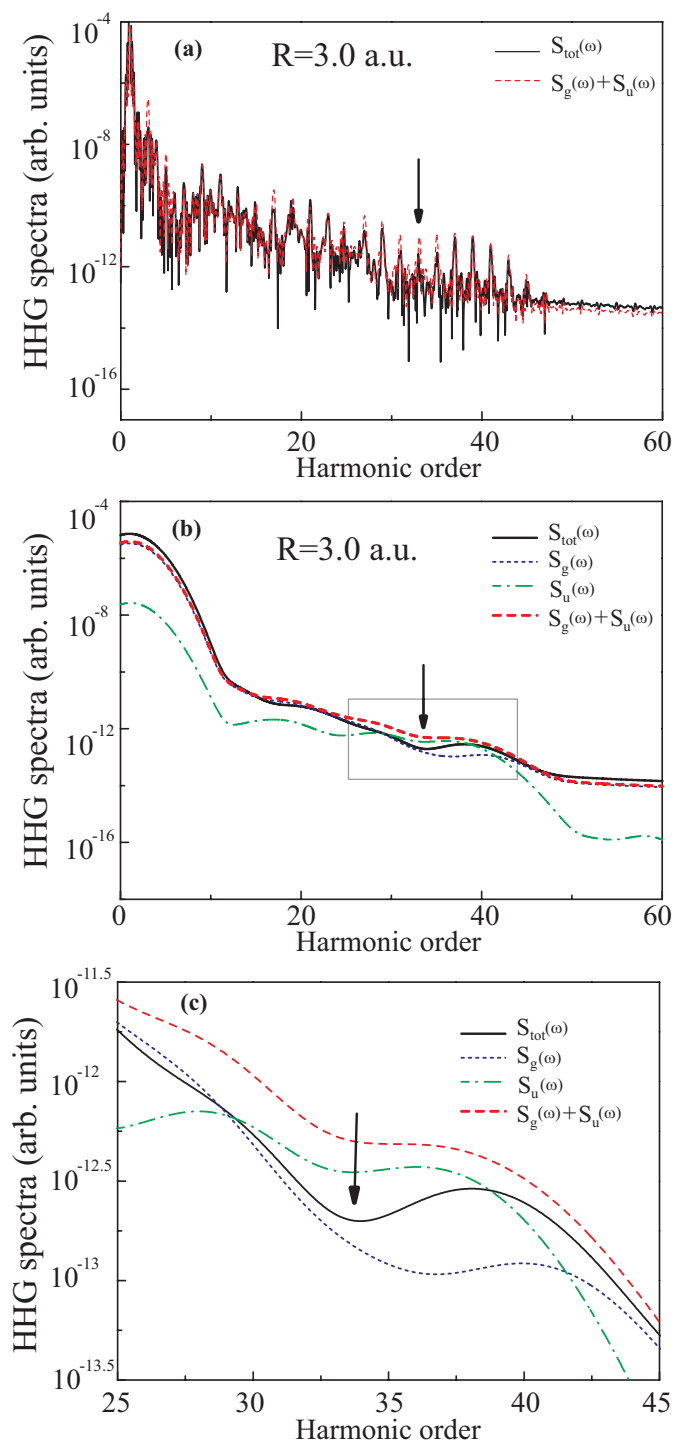

Figure 2. As figure 1, except that the internuclear separation has been increased to $R=3.0$ Bohr, the alignment angle changed to $\beta=55^{\circ}$ and the laser intensity decreased to $I=1.7 \times 10^{14} \mathrm{~W} / \mathrm{cm}^{2}$. The solid (black) arrow points to the orbital interference minimum in $S_{\text {tot }}(\omega)$.

should exhibit a minimum around $N_{\min }^{\mathrm{ti}}=29$. However, as shown by the dotted (blue) curve in figure 2(b), a minimum due to the two-center interference is not observed in $S_{\mathrm{g}}(\omega)$. The two-center interference model fails in this case, because the wave function $\psi_{\text {res }}(\boldsymbol{r}, t)$ in equation (5) is not accurately described by a plane wave, but contains effects of electronic structure in the bound and continuum parts of the spectrum [19]. It is clear from figure 2 that the minimum in the total HHG spectrum as pointed to by the solid (black) arrow is caused by the orbital interference. In figure 2(b), the magnitude of $S_{\mathrm{g}}(\omega)$ is comparable to $S_{\mathrm{u}}(\omega)$ for harmonic orders $25 \sim 40$, which enhances the orbital interference term and accordingly leads to destructive structure in $S_{\text {tot }}(\omega)$ for such a region. Although the two-center interference model fails in this case, the orbital 


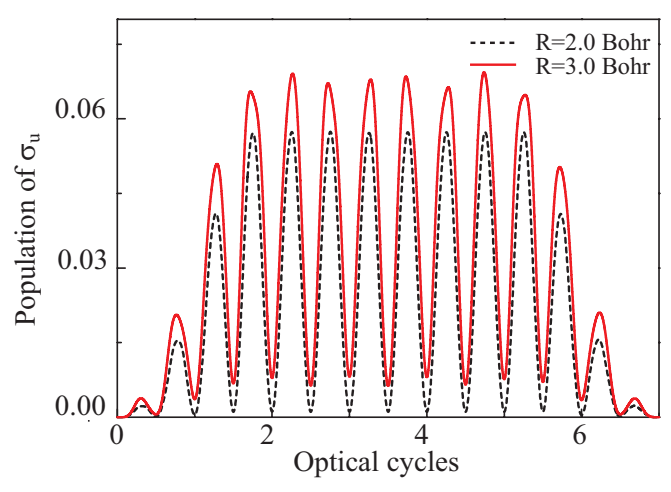

Figure 3. Time-dependent population of the $\sigma_{\mathrm{u}}$ first excited state for the cases of the laser parameters of figure 1 [dashed (black) curve] and figure 2 [solid (red) curve], respectively.

interference between the $\sigma_{\mathrm{g}}$ and $\sigma_{\mathrm{u}}$ states still forms a destructive structure in the total HHG spectrum, once the $\sigma_{\mathrm{g}}$ and $\sigma_{\mathrm{u}}$ components acquire comparable amplitudes.

\section{Summary and conclusion}

In summary, we demonstrated the important dynamical effects of an excited state in the HHG process, by calculating the HHG spectrum of an aligned $\mathrm{H}_{2}^{+}$molecule in linearly polarized laser fields. The 3D TDSE was solved for the electronic part, and the results show that the amplitudes describing HHG via the ground and the first excited state may interfere and give rise to a minimum when calculating the spectrum. At the equilibrium distance, due to the antisymmetric orbital structure of the $\sigma_{\mathrm{u}}$ state, the corresponding spectrum component $S_{\mathrm{u}}(\omega)$ exhibits a constructive interference structure at the order of the two-center interference minimum of $S_{\mathrm{g}}(\omega)$. Accordingly, the two-center interference minimum structure has been suppressed, and meanwhile, the orbital interference term has been enhanced since the recombination probabilities into the $\sigma_{\mathrm{g}}$ and $\sigma_{\mathrm{u}}$ states are comparable. The orbital interference forms a dip in the total HHG spectrum whose position is shifted slightly with respect to the prediction of the two-center interference formula. At larger internuclear distance, though the two-center interference model fails, the recombination probabilities into the two states may become comparable due to the strong coupling between the $\sigma_{\mathrm{g}}$ and $\sigma_{\mathrm{u}}$ states, which enhances the orbital interference and forms a minimum in the total HHG spectrum. The present results show that excited orbitals can play a role in the formation of distinct features, such as minima, in HHG spectra in addition to two-center interference $[1,2,13,14,18]$ and multi-electron dynamics [21,23-25].

\section{Acknowledgments}

LBM thanks J P Marangos for useful discussions. 


\section{References}

[1] Lein M, Hay N, Velotta R, Marangos J P and Knight P L 2002 Phys. Rev. Lett. 88183903

[2] Lein M, Hay N, Velotta R, Marangos J P and Knight P L 2002 Phys. Rev. A 66023805

[3] Lagmago Kamta G and Bandrauk A D 2004 Phys. Rev. A 70 011404(R)

[4] Lagmago Kamta G and Bandrauk A D 2005 Phys. Rev. A 71053407

[5] Le A-T, Tong X-M and Lin C D 2006 Phys. Rev. A 73041402

[6] Guhr M, McFarland B K, Farrell J P and Bucksbaum P H 2007 J. Phys. B: At. Mol. Phys. 40 3745

[7] Le A-T, Della Picca R, Fainstein P D, Telnov D A, Lein M and Lin C D 2008 J. Phys. B: At. Mol. Phys. 41081002

[8] Odzak S and Milosevic D B 2009 Phys. Rev. A 79023414

[9] Odzak S and Milosevic D B 2009 J. Phys. B: At. Mol. Phys. 42071001

[10] Chen Y J, Liu J and Hu B B 2009 Phys. Rev. A 79033405

[11] Le A-T, Lucchese RRand Lin C D 2009 J. Phys. B: At. Mol. Opt. Phys. 42211001

[12] Itatani J, Levesque J, Zeidler D, Niikura H, Pepin H, Kieffer J C, Corkum P B and Villeneuve D M 2004 Nature 432867

[13] Kanai T, Minemoto S and Sakai H 2005 Nature 435470

[14] Vozzi C, Calegari F, Benedetti E, Caumes J P, Sansone G, Stagira S, Nisoli M, Torres R, Heesel E, Kajumba N, Marangos J P, Altucci C and Velotta R 2005 Phys. Rev. Lett. 95153902

[15] McFarland B K, Farrell J P, Bucksbaum P H and Guhr M 2008 Science 3221232

[16] Zhou X B, Lock R, Li W, Wagner N, Murnane M M and Kapteyn H C 2008 Phys. Rev. Lett. 100 073902

[17] Baker S, Robinson J S, Lein M, Chirila C C, Torres R, Bandulet H C, Comtois D, Kieffer J C, Villeneuve D M, Tisch J W G and Marangos J P 2008 Phys. Rev. Lett. 101053901

[18] Boutu W, Haessler S, Merdji H, Breger P, Waters G, Stankiewicz M, Frasinski L J, Taieb R, Caillat J, Maquet A, Monchicourt P, Carre B and Salieres P 2008 Nature Physics 4545

[19] Wörner H J, Niikura H, Bertrand J B, Corkum P B and Villeneuve D M 2009 Phys. Rev. Lett. 102103901

[20] Mairesse Y, Levesque J, Dudovich N, Corkum P B and Villeneuve D M 2008 J. Mod. Opt. 552591

[21] Smirnova O, Mairesse Y, Patchkovskii S, Dudovich N, Villeneuve D M, Corkum P B and Ivanov M Y 2009 Nature 460972

[22] Smirnova O, Patchkovskii S, Mairesse Y, Dudovich N and Ivanov M Y 2009 Proc. Natl. Acad. Sci. USA 10616556

[23] Haessler S, Caillet J, Boutu W, Giovanetti-Teixeira, Ruchon T, Auguste T, Diveki Z, Breger P, Maquet A, Carre, Taieb R and Salieres P 2010 Nature Physics 6200

[24] Torres R, Siegel T, Brugnera L, Procino I, Underwood J G, Altucci A, Velotta R, Springate E, Froud C, Turcu I C E, Patchkovskii S, Ivanov M Yu, Smirnova O, and Marangos J P 2010 Phys. Rev. A $81051802(\mathrm{R})$

[25] Wörner H J, Bertrand J B, Hockett P, Corkum P B and Villeneuve D M 2010 Phys. Rev. Lett. 104233904

[26] Corkum P B 1993 Phys. Rev. Lett. 711994

[27] Lewenstein M, Balcou P, Ivanov M Y, L'Huillier A and Corkum P B 1994 Phys. Rev. A 492117

[28] Watson J B, Sanpera A, Chen X, and Burnett K 1996 Phys. Rev. A 53 R1962

[29] Figueira de Morisson Faria C and Augstein B B 2010 Phys. Rev. A 81043409

[30] Zhao Z, Yuan J and Brabec T 2007 Phys. Rev. A 76 031404(R)

[31] Sukiasyan S, McDonald C, Destefani C, Ivanov M Y and Brabec T 2009 Phys. Rev. Lett. 102 223002

[32] Kjeldsen T K, Nikolopoulos L A A and Madsen L B 2007 Phys. Rev. A 75063427

[33] Kjeldsen T K 2007 Wave packet dynamics studied by ab initio methods: Applications to strong-field ionization of atoms and molecules, Ph.D. thesis, Aarhus University, 
http://www.phys.au.dk/main/publications/PhD/Thomas_Kim_Kjeldsen.pdf

[34] Han Y C and Madsen L B 2010 Phys. Rev. A 81063430

[35] Burnett K, Reed V C, Cooper J and Knight P L 1992 Phys. Rev. A 453347

[36] Etches A, Madsen C B and Madsen L B 2010 Phys. Rev. A 81013409

[37] Bates D R 1951 J. Chem. Phys. 191122 